\section{RF11 SOCIAL EXCLUSION OF PEOPLE WITH MENTAL ILLNESS: THE WIDENING GAP FROM 1997 TO 2013 IN THE BELGIAN ADULT POPULATION}

P Smith*, P Nicaise, V Lorant. Institute of Health and Society, Université catholique de Louvain, Brussels, Belgium

\subsection{6/jech-2019-SSMabstracts. 126}

Background People with mental illness (MI) face issues of social integration such as finding a job and maintaining social relationships. In the past decades, many countries have developed specific social integration interventions and policies towards people with MI. Despite heterogeneous assessments of those specific interventions and policies, the social integration of people with MI and its improvement over time remains inconclusive. This study aimed to assess the evolution of the social integration of adults with moderate and severe $\mathrm{MI}$ in the general Belgian population between 1997 and 2013 .

Methods Data on the general adult population were retrieved from the Belgian Health Interview Survey in five cross-sectional waves (1997, 2001, 2004, 2008 and 2013, $\mathrm{n} \approx 10,000$ per wave). Three degrees of MI severity were compared using the General Health Questionnaire (GHQ-12): no MI, moderate MI, and severe MI (score $<4,4-7$, and $>7$ ). Indicators of social integration were the employment status, social contacts, and partnership situation. Age-and gender-adjusted prevalence of social integration indicators were estimated in the different periods. The evolution of prevalence was estimated with timetrend measures (absolute and relative change, and average annual percent chance). The association between social integration indicators and survey year was assessed with logistic regression models.

Results In 2013, the adjusted prevalence of social integration indicators of people without MI, with moderate MI, and with severe MI were respectively 46\%, 56\%, 69\% for unemployment, $2 \%, 6 \%, 11 \%$ for low social contacts, and 20\%, 25\%, $30 \%$ for being single. For unemployment and low social contacts, the gap between people with severe MI and the other two groups increased over time. The probability of being unemployed decreased significantly in 2013 compared to 1997 for people without $\mathrm{MI}(\mathrm{AOR}=0.59, \mathrm{CI} 95=0.53-0.65)$ and for people with moderate $\mathrm{MI}(\mathrm{AOR}=0.69, \mathrm{CI} 95=0.51-0.97)$, but not for people with severe $\mathrm{MI}(\mathrm{AOR}=1.08$, CI95 $=0.78-1.49)$. Conclusion The social exclusion gap between people with severe MI and people with moderate or without MI is widening over time. Employability and social support of people with more severe MI should be supported. Some sociodemographic determinants of people with severe MI may explain why their social integration has evolved differently over time.

\section{RF12 SUICIDE IDEATION AND MORTALITY RISK: POPULATION WIDE DATA LINKAGE STUDY}

${ }^{1}$ A Maguire, 'E Ross*, ${ }^{2} \mathrm{D}$ O'Hagan, 'D O'Reilly. 'Centre for Public Health, Queen's University Belfast, UK; ${ }^{2}$ Public Health Agency, Belfast, UK

10.1136/jech-2019-SSMabstracts.127

Rationale Very little is known about the association, if any, between suicide ideation, self-harm and completed suicide. Northern Ireland is unique in that it is the only country in the world to hold a registry of all presentations to Emergency Departments for suicide ideation. As NI has the highest suicide rate in the UK and Ireland it is vital to understand who is most at risk in order to target prevention strategies effectively. The aim of this study is to examine the characteristics of those individuals who present to Emergency Departments with suicide ideation and to explore the risk factors associated with subsequent suicide.

Data The Northern Ireland Registry of Self-Harm and Suicide Ideation contains information on all presentations to all Emergency Departments in NI for self-harm and suicide ideation. These data for the four years 2012-2015 were linked to centralised electronic data relating to primary care, social services and prescribed medication and mortality records.

Methods Descriptive analyses to explore the profile of those who present with suicide ideation and regression analyses to examine the likelihood of mortality post ideation after adjusting for a range of factors known to be associated with mental ill health.

Results The cohort consisted of all 1,483,435 individuals born or resident in NI from 1st January 1970 until 31st December 2015 (maximum age in 2015, 45 years). During the 4-year period 2012-2015, 3,644 (0.3\%) individuals presented with suicide ideation and $1,719(0.1 \%)$ individuals died by suicide. Ideation is more likely in men compared to women $(\mathrm{OR}=1.95,95 \% \mathrm{CI} 1.81,2.09)$ and in those aged 18-24 years. It is also associated with deprivation and with a history of being in care, with previous and current looked after children over 11 times more likely to present with suicide ideation compared to those not in care $(\mathrm{OR}=11.28$, 95\% CI 10.00,12.71). Of those who presented with ideation $1.13 \%$ subsequently died by suicide. After full adjustment, those who presented with suicide ideation were over 4 times more likely to die by suicide compared to those who did not $(\mathrm{OR}=4.52$, 95\%CI 3.29,6.21). Amongst suicide ideators there is no difference in likelihood of suicide based on age, gender or area of residence.

Further analysis is underway to explore which particular traits and characteristics of those who present with suicide ideation are most associated with risk of suicide and how this differs from those who self-harm in order in inform intervention targeting.

\section{Pregnancy \& Infant, Child \& Adolescent Health}

\section{RF13 MEASURING EXTRAUTERINE GROWTH RESTRICTION IN VERY PRETERM INFANTS: DOES CHOICE OF REFERENCE MATTER?}

${ }^{1}$ RER El Rafei*, ${ }^{1}$ PHJ Jarreau, ${ }^{2} \mathrm{JG}$ Gadzinowski, ${ }^{3} \mathrm{LD}$ Draper, ${ }^{4} \mathrm{MC}$ Cuttini, ${ }^{5} \mathrm{RM}$ Maier, ${ }^{1} \mathrm{JZ}$ Zeitlin. 'Obstetrical, Perinatal and Pediatric Epidemiology Research Team, INSERM U1153, Paris, France; ${ }^{2}$ Neonatology, Poznan University of Medical Sciences, Poznan, Poland; ${ }^{3}$ Health sciences, University of Leicester, Leicester, UK; ${ }^{4}$ Research unit of perinatal epidemiology, Bambino Gesu Children's hospital, Rome, Italy; ${ }^{5}$ Children's hospital, University hospital, Philipps university Marburg, Marburg, Germany

\subsection{6/jech-2019-SSMabstracts.128}

Background Extrauterine growth restriction (EUGR) among children born very preterm (VPT) is a risk factor for poor neurodevelopment. It is commonly defined as a weight for postmenstrual age (PMA) <10th percentile of postnatal growth references. Fenton's postnatal references, derived by meta-analysis of national birthweight and child growth charts, are commonly used in clinical care and research. Recently, the 
Intergrowth (IG) 21st project proposed alternative curves derived from multinational healthy preterm infants based on the hypothesis that normal VPT growth differs from term infants. We used these two approaches to investigate EUGR prevalence in a multinational sample of European VPT infants. Methods Data come from the EPICE (Effective Perinatal Intensive Care in Europe) project, an area-based study of infants born at less than 32 weeks' gestation in 2011/12 in 19 regions from 11 European countries. We included 6,471 infants discharged home before 50 weeks PMA. EUGR was defined as weight at discharge for PMA and sex $<10$ th percentile using Fenton and IG references. We compared the prevalence of EUGR by selected neonatal characteristics and country of birth, using X2 tests. We used generalized linear regression models with a Poisson distribution and robust standard errors to estimate adjusted risk ratios (aRR).

Results The prevalence of EUGR using Fenton's references was $43.9 \%$ for boys and $45.2 \%$ for girls (NS) compared to $33.6 \%$ for boys and $25.5 \%$ for girls for IG $(p<0.01)$. Prevalence of EUGR by country ranged from $24.7 \%$ in Sweden to $60.1 \%$ in Portugal for Fenton and from $14.0 \%$ in Sweden to $43.7 \%$ in Portugal for IG. Lower gestational age at birth, being small for gestational age at birth and having a severe neonatal morbidity were risk factors for being EUGR, regardless of the reference. Boys were more growth restricted than girls when using IG, but not Fenton. Adjusting for case-mix did not reduce variability between regions: the aRR for EUGR for Portuguese compared to Swedish VPT infants was $2.5(95 \%$ confidence interval (CI): 2.0-3.1) for Fenton and $3.3(95 \%$ CI: 2.6-4.6) for IG.

Conclusion Accurately identifying infants with sub-optimal growth is important for clinical care and for research on the etiology and consequences of EUGR. The difference in EUGR prevalence linked to choice of reference as well as the large variations between countries suggest that references should be validated in their target populations before adoption.

\section{RF14 CHANGES IN TRAJECTORIES FOR BLOOD PRESSURE AMONG CHINESE CHILDREN AND ADOLESCENTS: EVIDENCE FROM CHINA HEALTH AND NUTRITION SURVEY 1991-2011}

M Gao*, J Wells, L Li. GOS Institute of Child Health, University College London, London, UK

\subsection{6/jech-2019-SSMabstracts.129}

Background High blood pressure (BP) in adults is an important risk factor for cardiovascular disease (CVD) development and mortality. Childhood BP is not affected by antihypertension treatment, tracks into adulthood and is associated with early target organ disease. Previous reviews reported the varied trend of childhood BP between and within countries. Little is known whether BP trajectories during childhood have changed over time in developing countries with rapid economic development like China. As BP is strongly associated with body-size, we investigated whether BP trajectories have changed among Chinese children and adolescents and estimated the role of BMI and height trends to explain the change in BP trajectories during the past 2 decades.

Methods China Health and Nutrition Survey (CHNS), a mixed longitudinal household survey with eight waves from 1991 to 2011, was used to create four birth cohorts (children aged 7$17 \mathrm{y}$, born in 1981-85, 1986-90, 1991-95, 1996-2000,
$\mathrm{N}=\sim 16000$ ). Within each gender group, mixed effects cubic growth models were applied to estimate child-to-adolescent trajectories for systolic and diastolic BP (SBP and DBP) with and without adjustment for BMI and height by gender- and age-standardised $z$-scores. Between-cohort differences were examined by testing the interactions of each cohort with age terms.

Results Trajectories for SBP increased across cohorts: those for later-born cohorts tended to lay above early-born cohorts in both genders. After the adjustment of height and BMI, the differences in mean SBP reduced. The reduction was more evident in adolescence (vs childhood) and with adjustment for height (vs for BMI) trajectories.

For example, the difference between the last (born in 1996-2000) and first (born in 1981-85) cohorts was 1.90 $\mathrm{mmHg}$ (95\% CI: $0.54-3.26)$ for boys and $2.58 \mathrm{mmHg}(95 \%$ CI: 1.18-3.98) for girls at $7 \mathrm{y}$, and there was no difference at $16 y$ after adjustments. Similar patterns were seen for DBP.

Conclusion Our study is the first to investigate the change in BP trajectories during childhood in China. Later-born Chinese children and adolescents are characterized by higher BP than the earlier generations at the same age. This BP trend during recent decades was partly explained by more rapid growth in height and BMI in later-born children. Future study should exam other potential risk factors on BP trend in Chinese children.

\section{RF15 NATIONAL DATA OPT OUT PROGRAMME: CONSEQUENCES FOR MATERNAL AND CHILD HEALTH RESEARCH IN ENGLAND}

KM Lewis*, P Hardelid. Population, Policy and Practice, University College London, Great Ormond Street Institute of Child Health, London, UK

\subsection{6/jech-2019-SSMabstracts. 130}

Background The National Data Opt Out Programme was implemented in 2018 to enable users of the English NHS to electronically opt out of sharing their patient information for purposes other than their direct care. It has been reported that opt outs may affect the reliability of data used to evaluate services and conduct public health research; however, biases arising from opt outs have not previously been quantified. The aim of this study was to describe the extent to which rates of birth and maternity outcomes at Clinical Commissioning Group (CCG) level may be biased by patient opt outs.

Methods We selected one common and one rare maternity/ birth outcome: the rate of deliveries with caesarean sections and rate of births with very low birth weight. Average 2016 rates (per total number of deliveries/births) for both childbirth indicators are published online by Public health England. The percentage of total patients that have opted out as at 31 December 2018, by CCG, is publically available through NHS Digital. We simulated outcome rates across each CCG had opt outs not been applied to the data.

Results As at December 2018, the median CCG opt out rate across England was 2.4\%, ranging from a minimum of $0.3 \%$ to a maximum of $10.1 \%$. The average published proportion of deliveries with caesarean section was $27.3 \%$ (95\% CI 25.6, 29.1) and births with very low birth weight $1.18 \%$ (95\% CI $0.84,1.67)$. For the caesarean section indicator, our simulation produced an average minimum value of $26.6 \%$ and a 